\title{
Mortality, length of hospital stay, and nutritional status improvement in severely malnourished children with HIV infection in a tertiary center
}

\author{
Yoga Devaera
}

\begin{abstract}
Background Severe malnutrition contributes to child mortality rates, especially in children under five years of age. Concomitant HIV infection further increases the risk of death.

Objective To evaluate the impact of HIV infection on mortality, length of hospital stay and improvement of nutritional status in severely malnourished inpatients under five years of age.

Methods This retrospective study included children aged less than 5 years and admitted to a tertiary hospital for severe malnutrition. We excluded those with incomplete data, as well as those transferred to other hospitals or discharged against medical advice. Outcome measures were live/death status, length of hospital stay and improvement of nutritional status (weight gain and improved weight-for-height $Z$ score).

Results Of 104 eligible patients, 97 were included as study subjects. Their mean ages were 25.8 (SD 17.3) months for HIV-infected children and 16.3 (SD 15.3) months for HIVuninfected children. Death occurred in $31.8 \%$ and $18.7 \%$ of HIV-infected and uninfected subjects, respectively. Median length of hospital stay was 14.5 (range 5-51) days and 11 (range 1-99) days, respectively. There were no statistically significant differences in death rates or length of hospital stay between the two groups. Weight-for-height Z scores (WHZ) on admission and discharge in HIV-infected children were lower than those of uninfected children, but the weight gain (median weight gain of $0.45(-0.26$ to 1.9$) \mathrm{kg}$ vs $0.38(-0.81$ to 2.2$) \mathrm{kg}$ in HIV-infected and uninfected children, respectively) and improvement of WHZ [1 (SD 1.1) vs 0.9 (SD 1), respectively] were similar.

Conclusion Severely malnourished children with HIV infection had higher mortality rate, and longer hospitalizations than the uninfected group, although the differences were not statistically significant. They also had lower mean WHZ scores at admission and discharge, but nutritional improvement was
\end{abstract}

similar to those who were HIV-negative. [Paediatr Indones. 2012;52:362-6].

Keywords: protein energy malnutrition, mortality, HIV infection

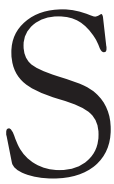

evere malnutrition contributes greatly to child mortality rates. The risk of death was reported to be 10 -fold higher in children with WHZ of $<-3$ (severe wasting), than in children with WHZ higher or equal to $-1 .{ }^{1}$ Severe malnutrition concomitant with HIV infection further increases the risk of mortality. A meta-analysis showed HIV prevalence to be high in children with severe acute malnutrition (SAM) in sub-Saharan Africa. HIVinfected children are at significantly increased risk of mortality. That study took place in an area of very high HIV-prevalence. ${ }^{2}$

From the Department of Child Health, University of Indonesia Medical School, Cipto Mangunkusumo Hospital, Jakarta, Indonesia.

Reprint requests to: Yoga Devaera, Division of Pediatric Nutrition and Metabolism, Department of Child Health, University of Indonesia Medical School, Cipto Mangunkusumo Hospital, Jl. Diponegoro No. 71, Jakarta 10430, Indonesia. Tel. +62-21-391 5179. E-mail: devaera@gmail.com 
Yoga Devaera: Mortality, length of hospital stay, and nutritional status improvement in HIV children

A Nigerian study with lower HIV prevalence (adult HIV prevalence of $0.8 \%$ ), showed that around $9 \%$ of children hospitalized for severe malnutrition were HIV-infected. ${ }^{3}$ In Indonesia, the prevalence of adult HIV was reported to be lower than $0.2 \%$, but the prevalence of pediatric HIV is increasing. In 2012, it was estimated that 4,860 Indonesian children live with HIV/AIDS. This number is expected to increase by greater than $25 \%$ in another 2 years. ${ }^{4}$ There have been few studies on severely malnourished children with HIV infection in Indonesia. We aimed to evaluate the impact of HIV infection on mortality, length of hospital stay, and nutritional improvement in severely malnourished children at a tertiary hospital.

\section{Methods}

This retrospective study was conducted at Cipto Mangunkusumo Hospital, a national referral hospital in Jakarta, Indonesia. Children aged less than 5 years and hospitalized with severe malnutrition from January 1 to December 31, 2011 were enrolled. Our hospital has several wards for infants and children, but only those admitted to the general pediatric ward were included. We also excluded those who were discharged against medical advice, transferred to other hospitals and those with incomplete data. Parental consent was not obtained since the study was retrospective and data was treated anonymously.

Subjects were weighed upon admission and daily thereafter until discharge, according to hospital procedure, using either an infant digital scale (SECA, Germany, maximum capacity $20 \mathrm{~kg}$ with 10-gram increments) or mechanical beam balance (SECA, Germany, maximum capacity $100 \mathrm{~kg}$ with 50 -g increments). Length or height was measured by length board or stadiometer (SECA, Germany). Ward nurses or residents in charge took these measurements.

Severe malnutrition was diagnosed based on clinical signs (e.g., wasting, baggy pants) and / or percentage of ideal body weight less than $70 \%$, according to the CDC growth chart 2000.5 Children with edema, organomegaly or conditions that masked actual weight were not included in the analysis involving weight gain. Treatment of severe malnutrition followed WHO guidelines, although several modifications occurred as instructed by pediatricians in charge. Criteria for discharge were stable condition, parental capability of feeding their child at home and a weight gain trend during the few days prior. We did not wait for the weight-for-height to rise above $70 \%$ to send children home, as we had many other children on our admission waiting list. Testing for HIV infection was not routinely done, but was based on clinical suspicions. HIV testing of children aged less than 18 months was done by polymerase chain reaction (PCR) test. Older children were tested for HIV by enzyme-linked immunosorbent assay (ELISA).

Duration of hospitalization was defined as the length of time from the date of admission to the date of discharge or death. Improvement of nutritional status was defined as Z-score increments between admission and discharge. We used WHZ from the WHO 2006 growth chart, instead of the CDC growth chart. Z-scores were calculated using WHO Anthro software. We also calculated subjects' weight gain between admission and discharge. Patients with conditions that masked actual weight such as edema, hepatomegaly, and hydrocephalus, were excluded from the analysis involving weight.

Data were extracted from the medical records and analyzed using SPSS version 15. All significance tests were two-sided and $\mathrm{P}$ values of $<0.05$ were considered to be statistically significant. Children's characteristics were compared using Student's t-test for continuous variables and Chi-square test for categorical variables or non-parametric tests, where applicable.

\section{Results}

Of 104 pediatric patients with severe malnutrition, 3 were discharged against medical advice, 1 was transferred to another hospital, and 3 had missing data, leaving 97 subjects to be analyzed. Of these, $23 \%$ of the subjects were HIV-positive. Others had medical conditions that could have caused severe malnutrition, such as gastrointestinal tract congenital malformations (atresia ani, morbus Hirshprung's), congenital heart diseases, chronic 
Yoga Devaera: Mortality, length of hospital stay, and nutritional status improvement in HIV children

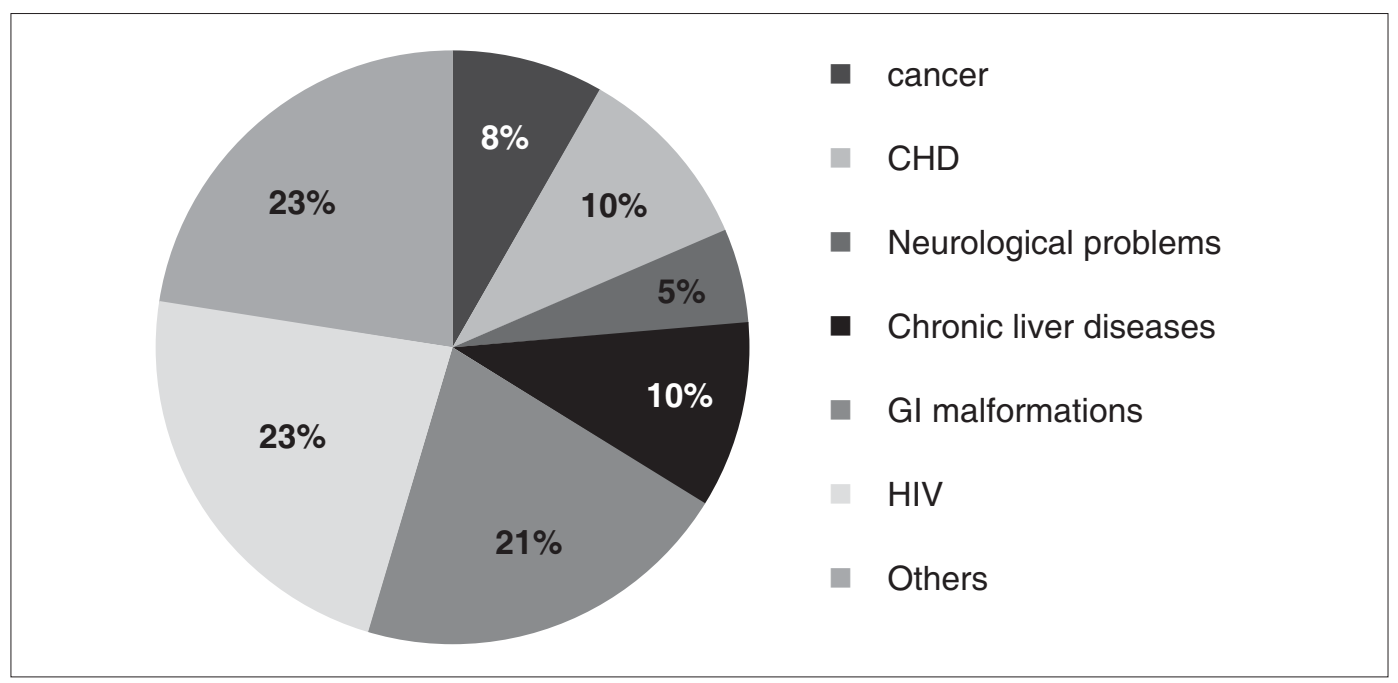

Figure 1. Distribution medical conditions in subjects with severe malnutrition

Table 1. Characteristics of subjects

\begin{tabular}{lcc}
\hline Characteristics & HIV-positive & HIV-negative \\
& $\mathrm{n}=22$ & $\mathrm{n}=75$ \\
\hline Mean age, months (SD) & $25.8(17.3)$ & $16.3(15.3)$ \\
Male gender, $\mathrm{n}$ & 14 & 43 \\
Mean WHZ on admission, score (SD) & $-4.7(1.6)$ & $-3.5(2)$ \\
\hline
\end{tabular}

Table 2. Nutritional improvement of subjects by group

\begin{tabular}{|c|c|c|c|}
\hline Variables & $\begin{array}{l}\text { HIV-positive } \\
\quad(n=22)\end{array}$ & $\begin{array}{l}\text { HIV-negative } \\
\qquad(n=58)\end{array}$ & $P$ value \\
\hline Mean weight gain, $\mathrm{kg}$ (range) & $0.45(-0.26$ to 1.9$)$ & $0.38(-0.81$ to 2.2$)$ & 0.212 \\
\hline Mean WHZ at time of discharge, score (SD) & $-3.7(1.9)$ & $-2.6(2.1)$ & 0.037 \\
\hline Mean WHZ improvement, score (SD) & $1 \quad(1.1)$ & $0.9(1.1)$ & 0.722 \\
\hline
\end{tabular}

Mann-Whitney $U$ test

Table 3. Subjects' outcomes by group

\begin{tabular}{llll}
\hline Outcomes & $\begin{array}{c}\text { HIV-positive } \\
(\mathrm{n}=22)\end{array}$ & $\begin{array}{c}\text { HIV-negative } \\
(\mathrm{n}=75)\end{array}$ & P value \\
\hline Death, \% & 31.8 & 18.7 & $0.239^{*}$ \\
Mean length of stay, days (range) & 14.5 (5 to 51$)$ & 11 (1 to 99$)$ & $0.101+$ \\
\hline
\end{tabular}

${ }^{*}$ Fisher's exact test; + Mann-Whitney $\mathrm{U}$ test

liver diseases, neurological problems, cancer and concurrent infectious diseases (pneumonia and chronic diarrhea). Figure 1 shows the distribution of the medical conditions observed in our subjects with severe malnutrition.

The HIV-positive subjects tended to be older and more severely malnourished. They had a lower weight and WHZ on admission (Table 1). However, the mean weight gain and improvement of WHZ during hospitalization was not significantly different between the two groups (Table 2). HIVpositive subjects also had higher mortality and length of hospital stay, but these were similarly not significantly different from the HIV-negative group (Table 3). Further analysis, in which we excluded one patient in the HIV-negative group with an unusually long hospital stay of 99 days, did not change the significance. 
Yoga Devaera: Mortality, length of hospital stay, and nutritional status improvement in HIV children

\section{Discussion}

This study is one of the first in Indonesia to report on the characteristics and outcomes of children hospitalized with severe malnutrition and HIV infection. Although we did not routinely screen for HIV infection in severely malnourished children in this study, we found quite a high rate of HIV infection. More than one-fifth of children aged under-five years hospitalized for severe malnutrition were infected with HIV. This number was higher than that reported in African countries. A 1997 Nigerian study reported an HIV prevalence of $1.9 \%,{ }^{6}$ while a 1993 study in Burkina Faso study reported an HIV prevalence of $14.0 \% .{ }^{7}$ Our higher rate of HIV infection may be due to our status as a national referral hospital, in which we would expect to have a high burden of serious diseases. We also found that more than half of our subjects had predisposing factors for malnutrition. Subjects' underlying medical conditions were similar to causes of severe malnutrition in developed countries, except for HIV infection. This difference contrasted with the African studies where primary malnutrition was a major cause. ${ }^{8}$

Patients with HIV infection were older than those without HIV, but more severely malnourished. The HIV-negative subjects had mainly congenital diseases that manifested early in life, and had better WHZ scores at admission. At discharge, patients without HIV infection also had better nutritional status. But during hospitalization, there were no significant differences in weight gain and WHZ score improvement between the two groups. Similarly, a Malawi study showed that HIV-infected patients who survived had similar nutritional recovery to the non-HIV-infected patients. ${ }^{9}$ A limitation of our study was that we did not evaluate the nutritional regimen given to the two groups.

The overall mortality rate for severely malnourished children in our hospital was $22 \%$. More HIV-infected patients died, but mortality was not significantly different from the HIV-negative group. Also, the mean length of hospital stay between the two groups was not significantly different. This observation may be explained by our status as a national referral center where we deal with many complex cases. Our patients' characteristics were different from those of studies at nutritional rehabilitation centers. They also found the mortality rate to be higher in HIV-infected groups.., 10

Severely malnourished children with HIV infection had higher mortality rates and longer hospitalizations, although the differences were not statistically significant from uninfected subjects. HIV-positive subjects had lower mean WHZ score at admission, but their nutritional improvement was similar to those who were HIV-negative. Further study needs to be done to identify risk factors for those who did not survive, in order to improve the management of children with severe malnutrition.

\section{References}

1. Peletier DL, Frongillo EA Jr, Schroeder DG, Habicht JP. The effects of malnutrition on child mortality in developing countries. Bull World Health Org. 1995;73:443-8.

2. Fergusson P, Tomkins A. HIV prevalence and mortality among children undergoing treatment for severe acute malnutrition in sub-Saharan Africa: a systematic review and meta-analysis. Trans R Soc Trop Med Hyg. 2009;103:541-8.

3. Madec Y, Germanaud D, Moya-Alvarez V, Alkassoum W, Issa A, Amadou M, Tchiombiano S, et al. HIV prevalence and impact on renutrition in children hospitalised for severe malnutrition in Niger: an argument for more systematic screening. PLoS One. 2011;6:e22787.

4. Direktorat Jendral Pengendalian Penyakit dan Penyehatan Lingkungan Kementrian Kesehatan RI. Situasi terkini HIV/ AIDS. [cited 2012 May 13]. Available from: http://www.pppl. depkes.go.id/_asset/_download/SITUASI_AIDS_TERKINI. $p d f ? v m=r \varepsilon v m=r$

5. Department of Health and Human Services, Center for Disease Control and Prevention, National Center for Health Statistics. 2000 CDC growth chart for the United States: Methods and development. US Government Printing Office: Washington; 2002.

6. Akenami FO, Koskiniemi M, Ekanem EE, Bolarin DM, Vaheri A. Seroprevalence and coprevalence of HIV and HBsAg in Nigerian children with/without protein energy malnutrition. Acta Trop. 1997;64:167-74.

7. Prazuck T, Tall F, Nacro B, Rochereau A, Traore A, Sanou T, et al. HIV infection and severe malnutrition: a clinical and epidemiological study in Burkina Faso. AIDS. 1993; 7:103-8.

8. Grover Z, Ee LC. Protein energy malnutrition. Pediatr Clin N Am. 2009;56:1055-68. 
Yoga Devaera: Mortality, length of hospital stay, and nutritional status improvement in HIV children

9. Fergusson P, Chinkhumba J, Grijalva-Eternod C, Banda T, Mkangama C, Tomkins A. Nutritional recovery in HIV-infected and HIV-uninfected children with severe acute malnutrition. Arch Dis Child. 2009;94:512-6.
10. Chinkhumba J, Tomkins A, Banda T, Mkangama C, Fergusson P. The impact of HIV on mortality during inpatient rehabilitation of severely malnourished children in Malawi. Trans R Soc Trop Med Hyg. 2008;102:639-44. 